\section{Emergence of Carbapenem-Resistant Acinetobacter baumannii in Nursing Homes With High Background Rates of MRSA Colonization}

Vincent C. C. Cheng, MD; ${ }^{1,2}$ Jonathan H. K. Chen, $\mathrm{PhD} ;^{1}$ W. C. Ng, MBA(HSM); Janet Y. H. Wong, PhD; ${ }^{4}$ Denise M. K. Chow, PhD; ${ }^{4}$ T. C. Law, MSc(IC); ${ }^{3}$ Simon Y. C. So, MMedSc; ${ }^{1}$ Sally C. Y. Wong, MD; ${ }^{1}$ T. C. Chan, MD; ${ }^{3}$ Felix H. W. Chan, $\mathrm{MD} ;{ }^{3}$ P. L. Ho, MD; ${ }^{1}$ K. Y. Yuen, $\mathrm{MD}^{1}$

Carbapenem-resistant Acinetobacter baumannii (CRAB) with diverse multilocus sequence typing emerged among our nursing home residents $(6.5 \%)$ with a high background rate of MRSA (32.2\%). Rectal swabs yielded a higher rate of CRAB detection than axillary or nasal swabs. Bed-bound status, use of adult diapers, and nasogastric tube were risk factors for CRAB colonization.

Infect Control Hosp Epidemiol 2016;37:983-986

Transmission of multidrug-resistant organisms (MDROs) has been increasingly reported in nursing homes. ${ }^{1,2}$ In Hong Kong, the prevalence of MRSA in nursing homes increased from $2.8 \%$ to $21.6 \%$ between 2005 and 2011. ${ }^{3,4}$ While infection control measures are generally more stringent in acute hospitals than in nursing homes, the latter have become epidemic centers for MDROs. With the emergence of carbapenem-resistant gram negative organisms in our hospitals, ${ }^{5,6}$ it is important to determine the prevalence of and risk factors for carbapenem-resistant Acinetobacter baumannii (CRAB), carbapenemase-producing Enterobacteriaceae (CPE), and other MDROs in our nursing homes including methicillin-resistant Staphylococcus aureus (MRSA) and vancomycin-resistant Enterococci (VRE). These findings are useful in the formulation of infection control policy.

\section{METHODS}

This study was conducted from July 1, 2015, to August 31, 2015, to determine the prevalence of MDROs among 28 nursing homes in the Hong Kong West District with a population of 0.53 million. The role of nursing homes was described previously. ${ }^{4}$ Trained research nurses collected nasal swabs, axillary swabs, and rectal swabs from consented subjects all on the same day according to a standard protocol. The specimens were tested by the microbiology laboratory for the presence of CRAB, CPE, MRSA, and VRE as previously described. ${ }^{4-6}$ The bacterial species were confirmed by matrix-assisted laser desorption/ionization time-of-flight mass spectrometry (MALDI-TOF MS; Bruker Daltonics, Bremen, Germany). Susceptibility of the isolates was determined using the Kirby-Bauer disc diffusion method or E test, and the results were interpreted according to the guidelines of the Clinical and Laboratory Standards Institute (CLSI). For molecular typing of CRAB and MRSA, multilocus sequencing typing (MLST) and spa typing were performed on isolates of CRAB and MRSA respectively. ${ }^{6,7}$ Patient demographic data were retrieved and analyzed. The $\chi^{2}$ test and Student $t$ test were used as appropriate with SPSS version 20 (IBM, Armonk, NY). Factors with $P<.05$ in univariate analysis were chosen for multivariate analysis. A final model was selected using a forward selection procedure. This study was approved by our institutional review board.

\section{RES ULTS}

During the study period, 1,408 (60.6\%) of 2,322 subjects from 28 nursing homes consented to undergo screening. The mean age was $83.8 \pm 10.3$ years and $854(60.7 \%)$ were female. Of 691 $(49.1 \%)$ residents who were functionally dependent, 474 of $691(68.6 \%)$ were chair bound and 217 of $691(31.4 \%)$ were bed bound. Of 1,408 residents, 909 (64.6\%) were incontinent requiring the use of adult diapers; $62(4.4 \%)$ had sacral sores; $71(5.0 \%)$ had nasogastric tubes; $4(0.3 \%)$ had percutaneous gastrostomy tubes; $4(0.3 \%)$ had Tenckhoff catheters; and $75(5.3 \%)$ had long-term urinary catheters.

Overall, colonization of MDROs was found in 494 of 1,408 residents (35.1\%). MRSA colonization in any 1 of the 3 swabs was found in 454 residents (32.2\%), while CRAB colonization was found in 92 residents (6.5\%). A single resident had CPE with NDM-1-producing E. coli. VRE was not detected in any screened specimens. Of the 1,408 residents, 399 (28.3\%) were colonized with MRSA only; 37 (2.6\%) were colonized with CRAB only; and 55 (3.9\%) were co-colonized with MRSA and CRAB. The subsequent analyses on residents focused on CRAB and/or MRSA colonization because the number of residents with CPE was small. The prevalence of CRAB and MRSA colonization among residents through different body sites is summarized in Table 1.

The univariate and multivariate analyses of the epidemiological risk factors for CRAB and MRSA colonization among residents in nursing homes are shown in Tables 2 and 3, respectively. Bed-bound status, incontinence with the use of adult diapers, and presence of a nasogastric tube were the common risk factors for both CRAB and MRSA colonization by multivariate analysis.

Molecular characterization of CRAB and MRSA was performed in 25 and 30 non-duplicated patient isolates from 6 nursing homes and showed a high prevalence of CRAB or MRSA respectively. A predominant spa type of MRSA t1081 was identified in 19 of 30 MRSA isolates (63.3\%), in 
TABLE 1. Prevalence of Carbapenem-Resistant Acinetobacter baumannii (CRAB) and Methicillin-Resistant Staphylococcus aureus (MRSA) Colonization Among Residents in Nursing Homes by Sampling of Different Body Sites

\begin{tabular}{lcc}
\hline Body Site & $\begin{array}{c}\text { Prevalence of } \\
\text { CRAB } \\
\text { Colonization, No. } \\
(\%)(\mathrm{N}=1,408)\end{array}$ & $\begin{array}{c}\text { Prevalence of } \\
\text { MRSA } \\
\text { Colonization, No. } \\
(\%)(\mathrm{N}=1,408)\end{array}$ \\
\hline Nasal swab & $14(1.0)^{\mathrm{a}}$ & $265(18.8)^{\mathrm{b}}$ \\
Axillary swab & $34(2.4)^{\mathrm{a}}$ & $129(9.2)^{\mathrm{b}}$ \\
Rectal swab & $64(4.5)^{\mathrm{a}}$ & $259(18.4)^{\mathrm{b}}$ \\
Combination of any 2 sites & & \\
Nasal and/or axillary swabs & $46(3.3)$ & $336(23.9)$ \\
Rectal and/or axillary swabs & $83(5.9)$ & $315(22.4)$ \\
Rectal and/or nasal swabs & $73(5.2)$ & $418(29.7)$ \\
Any one site & $92(6.5)$ & $454(32.2)$ \\
\hline
\end{tabular}

${ }^{\mathrm{a}}$ The prevalence of CRAB colonization was statistically significant by sampling of different body sites $(P<.001)$.

${ }^{\mathrm{b}}$ The prevalence of MRSA colonization was statistically significant by sampling of different body sites $(P<.001)$. keeping with the predominant MRSA clone of ST45/spa t1081 in our locality. ${ }^{7}$ The MLST patterns of CRAB were diverse, including 8 of 25 clones of ST575 (32.0\%), and 7 newly reported MLST patterns in 7 patients with gastrointestinal colonization of CRAB.

\section{I S C US S I ON}

In this study, we have demonstrated the emergence of CRAB colonization and a high prevalence of MRSA among nursing homes residents in Hong Kong. The prevalence of CRAB colonization was $6.5 \%$, almost 3 times higher than in hospitalized patients identified through active surveillance culture in a previous study. ${ }^{8}$ The rate of CRAB was highest among rectal swab cultures, which coincides with our previous finding that residents in nursing homes have the most significant risk factor for gastrointestinal colonization of CRAB among hospitalized patients. ${ }^{8}$ In our study, the burden of antimicrobial-resistant $A$. baumannii was comparatively less

TABLE 2. Epidemiological Characteristics of Residents With or Without Colonization by Methicillin-Resistant Staphylococcus aureus (MRSA) and Carbapenem-Resistant Acinetobacter baumannii (CRAB)

\begin{tabular}{|c|c|c|c|c|c|}
\hline & $\begin{array}{l}\text { (A) Patients Without } \\
\text { Colonization by any MDROs, } \\
\text { No. }(\%)(\mathrm{N}=914)^{\mathrm{a}}\end{array}$ & $\begin{array}{l}\text { (B) Patients With } \\
\text { Colonization by } \\
\text { CRAB, No. (\%) } \\
\quad(\mathrm{N}=92)\end{array}$ & $\begin{array}{l}P \text { Value }(\mathrm{A}) \\
\text { vs }(\mathrm{B})\end{array}$ & $\begin{array}{l}\text { (C) Patients With } \\
\text { Colonization by } \\
\text { MRSA, No. (\%) } \\
(\mathrm{N}=454)\end{array}$ & $\begin{array}{l}P \text { Value }(\mathrm{A}) \\
\text { vs }(\mathrm{C})\end{array}$ \\
\hline Age (mean years $\pm S D)$ & $83.6 \pm 10.2$ & $81.8 \pm 11.2$ & .107 & $84.5 \pm 10.0$ & .111 \\
\hline Male sex & $358(39.2)$ & $56(60.9)$ & $<.001$ & $170(37.4)$ & .827 \\
\hline Dependency: chair-bound & $277(30.3)$ & $42(45.7)$ & .003 & $177(39.0)$ & .001 \\
\hline $\begin{array}{l}\text { Presence of sacral sore } \\
\text { Presence of indwelling device }\end{array}$ & $25(2.7)$ & $12(13.0)$ & $<.001$ & $31(6.8)$ & .001 \\
\hline Nasogastric tube & $18(2.0)$ & $14(15.2)$ & $<.001$ & $48(10.6)$ & $<.001$ \\
\hline Percutaneous gastrostomy tube & $1(0.1)$ & $1(1.1)$ & .127 & $3(0.7)$ & .161 \\
\hline $\begin{array}{l}\text { Urinary catheter } \\
\text { Underlying diseases }\end{array}$ & $30(3.3)$ & $7(7.6)$ & .036 & $42(9.3)$ & $<.001$ \\
\hline Chronic renal failure & $26(2.8)$ & $6(6.5)$ & .055 & $25(5.5)$ & .014 \\
\hline Liver cirrhosis & $13(1.4)$ & $1(1.1)$ & .794 & $4(0.9)$ & .395 \\
\hline Diabetes mellitus & $74(8.1)$ & $15(16.3)$ & .008 & $52(11.5)$ & .043 \\
\hline $\begin{array}{l}\text { Malignancies } \\
\text { Use of antibiotic in preceding }\end{array}$ & 6 months $27(3.0)$ & $7(7.6)$ & .019 & $22(4.8)$ & .076 \\
\hline $\begin{array}{l}\text { Beta-lactam/beta-lactamase } \\
\text { inhibitors }\end{array}$ & $33(3.6)$ & $15(16.3)$ & $<.001$ & $50(11.0)$ & $<.001$ \\
\hline Cephalosporins & $10(1.1)$ & $2(2.2)$ & .363 & $11(2.4)$ & .060 \\
\hline Carbapenems & $12(1.3)$ & $4(4.3)$ & .027 & $17(3.7)$ & .003 \\
\hline Fluoroquinolones & $29(3.2)$ & $10(10.9)$ & $<.001$ & $29(6.4)$ & .005 \\
\hline $\begin{array}{l}\text { History of hospitalization in the } \\
\text { past } 6 \text { months }\end{array}$ & $282(30.9)$ & $52(56.5)$ & $<.001$ & $220(48.5)$ & $<.001$ \\
\hline
\end{tabular}

${ }^{a}$ MDROs include methicillin-resistant Staphylococcus aureus, carbapenem-resistant Acinetobacter baumannii and carbapenemase-producing Enterobacteriaceae. 
TABLE 3. Risk Factors in Residents With Colonization by Carbapenem-Resistant Acinetobacter baumannii (CRAB) and MethicillinResistant Staphylococcus aureus (MRSA) Using Multiple Logistic Regression ${ }^{\text {a }}$

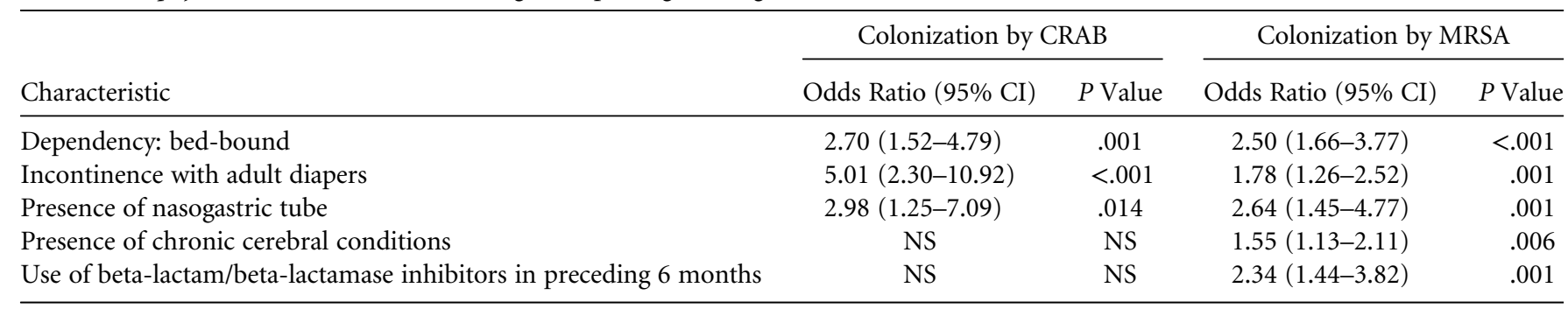

NOTE. CI, confident interval.

${ }^{a}$ All epidemiological characteristics listed were used as independent variables. Final model was selected using a forward selection procedure. For the analysis of CRAB colonization, the Hosmer-Lemeshow goodness-of-fit statistic was 4.17 , and $P=.841$. For the analysis of MRSA colonization, the Hosmer-Lemeshow goodness-of-fit statistic was 3.23, and $P=.780$.

than that in the United States, where 15\% of high-risk nursing home residents have been reported to be colonized with multidrug-resistant $A$. baumannii (MRAB) using active surveillance culture. ${ }^{2}$ Functional disability was an important risk factor for MRAB colonization, ${ }^{2}$ and we further demonstrated that physical dependency such as bed-bound status, incontinence requiring adult diapers, and nasogastric tube feeding were significant risk factors for $\mathrm{CRAB}$ colonization in our multivariate analysis. Unlike MRSA, for which a predominant spa type prevailed, CRAB isolated from nursing home residents had diverse MLST patterns. This finding contrasts with the observation of clonal dissemination of $A$. baumannii in hospitals, ${ }^{6}$ suggesting that a successful clone of A. baumannii has not yet been established in these nursing homes.

In this study, the prevalence of MRSA colonization among elderly residents in nursing homes was $32 \%$, which was 10 times higher than the prevalence reported in Hong Kong 10 years ago. ${ }^{3}$ The predominant spa type of MRSA was t1081, which was also the most commonly identified spa type among the hospital isolates. ${ }^{7}$ Notably, the rate of gastrointestinal colonization of MRSA was as high as nasal colonization among our residents. Widespread environmental contamination had been shown previously in patients with MRSA colonization of the gastrointestinal tract. ${ }^{9}$ This factor may explain why MRSA in nursing homes could not be brought under control.

With the emergence of CRAB and increasing prevalence of MRSA colonization in our nursing homes, it is important to enhance infection control interventions. Active surveillance culture may facilitate early recognition of residents colonized with MDROs. In our analysis, collection of nasal swabs and rectal swabs, even without axillary swabs, detected $80 \%$ and $92 \%$ of CRAB and MRSA, respectively. Hand hygiene by healthcare workers (HCWs) in nursing homes should be enforced. In particular, supervisors of nursing homes should directly observe and ensure the implementation of hand hygiene practices by HCWs during and after adult diaper changes and feeding via nasogastric tubes because these were significant risk factors for CRAB and MRSA colonization in our study. Additionally, directly observed hand hygiene before meals and oral medication administration by conscious residents should be promoted to prevent ingestion of MDROs into the gastrointestinal tract. ${ }^{6,10}$

We are aware of several limitations in this study. First, we did not screen patients who were demented or mentally incapacitated and had not undergone interval screening. Both of these factors may have led to the underestimation of MDRO prevalence. Second, we did not assess the degree of environmental contamination, and gastrointestinal colonization of MRSA was associated with significant environmental contamination. ${ }^{9}$ Third, we selected CRAB and MRSA strains from nursing homes with high incidence, which may have led to the overrepresentation of "epidemic clones." However, our findings highlight the rapid emergence of CRAB in nursing homes, which deserves the attention of infection control professionals.

\section{ACKNOWLEDGMENTS}

Financial support: This study was partially supported by the Health and Medical Research Fund (HMRF), Food and Health Bureau, Hong Kong SAR Government (grant no. HKM-15-M12).

Potential conflicts of interest: All authors report no conflicts of interest relevant to this article.

Affiliations: 1. Department of Microbiology, Queen Mary Hospital, Hong Kong Special Administrative Region, China; 2. Infection Control Team, Queen Mary Hospital, Hong Kong Special Administrative Region, China; 3. Community Geriatric Assessment Team, Fung Yiu King Hospital, Hong Kong Special Administrative Region, China; 4. School of Nursing, The University of Hong Kong, Hong Kong Special Administrative Region, China.

Address correspondence to KY Yuen, Department of Microbiology, Queen Mary Hospital, Hong Kong Special Administrative Region, China (kyyuen@hku.hk).

Received December 10, 2015; accepted March 11, 2016; electronically published April 25, 2016

(C) 2016 by The Society for Healthcare Epidemiology of America. All rights reserved. 0899-823X/2016/3708-0017. DOI: 10.1017/ice.2016.84

\section{REFERENCES}

1. D’Agata EM, Habtemariam D, Mitchell S. Multidrug-resistant Gram-negative bacteria: inter- and intradissemination among 
nursing homes of residents with advanced dementia. Infect Control Hosp Epidemiol 2015;36:930-935.

2. Mody L, Gibson KE, Horcher A, et al. Prevalence of and risk factors for multidrug-resistant Acinetobacter baumannii colonization among high-risk nursing home residents. Infect Control Hosp Epidemiol 2015;36:1155-1162.

3. Ho PL, Wang TK, Ching P, et al. Epidemiology and genetic diversity of methicillin-resistant Staphylococcus aureus strains in residential care homes for elderly persons in Hong Kong. Infect Control Hosp Epidemiol 2007;28:671-678.

4. Cheng VC, Tai JW, Wong ZS, et al. Transmission of methicillinresistant Staphylococcus aureus in the long-term care facilities in Hong Kong. BMC Infect Dis 2013;13:205.

5. Ho PL, Cheung YY, Wang Y, et al. Characterization of carbapenem-resistant Escherichia coli and Klebsiella pneumoniae from a healthcare region in Hong Kong. Eur J Clin Microbiol Infect Dis Jan 6 2016, [Epub ahead of print].

6. Cheng VC, Chen JH, Poon RW, et al. Control of hospital endemicity of multiple-drug-resistant Acinetobacter baumannii
ST457 with directly observed hand hygiene. Eur J Clin Microbiol Infect Dis 2015;34:713-718.

7. Gruteke P, Ho PL, Haenen A, Lo WU, Lin CH, de Neeling AJ. MRSA spa t1081, a highly transmissible strain endemic to Hong Kong, China, in the Netherlands. Emerg Infect Dis 2015;21:1074-1076.

8. Cheng VC, Chen JH, So SY, et al. Use of fluoroquinolones is the single most important risk factor for the high bacterial load in patients with nasal and gastrointestinal colonization by multidrug-resistant Acinetobacter baumannii. Eur J Clin Microbiol Infect Dis 2015;34:2359-2366.

9. Boyce JM, Havill NL, Otter JA, Adams NM. Widespread environmental contamination associated with patients with diarrhea and methicillin-resistant Staphylococcus aureus colonization of the gastrointestinal tract. Infect Control Hosp Epidemiol 2007;28:1142-1147.

10. Cheng VC, Tai JW, Wong LM, et al. Effect of proactive infection control measures on benchmarked rate of hospital outbreaks: An analysis of public hospitals in Hong Kong over 5 years. Am J Infect Control Jun 6 2015, [Epub ahead of print]. 